\title{
Granulomatous Mastitis: Incidence and Experience in a Mexican Institute
}

\author{
Arnoldo Topete ${ }^{1 *}$, Olin Carrasco' ${ }^{1}$, Gabriel Barrera ${ }^{1}$, Juan Garzón1, Eduardo Gutierrez ${ }^{1}$, \\ Cuahutémoc Márquez¹, Rodrigo Dávila1, Marco Cuervo', María Irene Rivera² \\ ${ }^{1}$ Department of Plastic and Reconstructive Surgery, Pemex Hospital, Mexico City, Mexico \\ ${ }^{2}$ Department of Pathological Anatomy, Pemex Hospital, Mexico City, Mexico \\ Email: *arnoldo.topete@gmail.com, plastiksurg@icloud.com, gabrielbarrera.md@gmail.com, garzonmuvdi@gmal.com, \\ jorge.eduardo.gutierrez@pemex.com,cauhutemoc.marquez@pemex.com,drrodrigodavila@yahoo.com.mx,macv_61@hotmail.com
}

How to cite this paper: Topete, A., Carrasco, O., Barrera, G., Garzón, J., Gutierrez, E., Márquez, C., Dávila, R., Cuervo, M. and Rivera, M.I. (2019) Granulomatous Mastitis: Incidence and Experience in a Mexican Institute. Journal of Biosciences and Medicines, 7, 24-32.

https://doi.org/10.4236/jbm.2019.76003

Received: April 25, 2019

Accepted: June 1, 2019

Published: June 4, 2019

Copyright $\odot 2019$ by author(s) and Scientific Research Publishing Inc. This work is licensed under the Creative Commons Attribution International License (CC BY 4.0).

http://creativecommons.org/licenses/by/4.0/

(c) (i) Open Access

\begin{abstract}
The significance of this work is that chronic granulomatous disease can mimic two common breast diseases; breast cancer and breast abscess; so it is mandatory that the diagnosis is made in order to avoid mistaken carcinoma diagnosis. On the other hand, the current treatment of this particular disease still remains controversial. Objectives: The aim of this study is to expose and describe the chronicity and the impact in a patient's quality of life with Granulomatous Mastitis diagnosis. We describe the evolution of the disease and the treatment of the disease in a Mexican Institution. Material and methods: In a descriptive retrospective study we identified 9 patients with idiopathic granulomatous mastitis (IMG), all of them were diagnosed with histopathological findings. Results: In a period of time between January 2005 and December 2017, we included a total of 9 female patients. All of them have had histopathological findings consistent with granulomatous mastitis. The age range was 25 - 83 years old (mean 50.8 yrs). In 4 patients (44.4\%) had the right side affected, three patients (33.3\%) had the left side affected and 2 patients (22.2\%) had a bilateral presentation. In 3 patients (33.3\%) we observed more than one affected quadrant and 4 patients (44.4\%) had a diffuse presentation on image. The most common symptom was breast pain in 9 patients (100\%) and the most common sign was the presence of a nodule, 8 patients (88.8\%). We observed erythema in 7 cases (77.7\%) followed by abscess, edema and pus secretion in 6 patients $(66.6 \%)$. The most frequent treatment was the use of a combination of NAIDs and antibiotic in 8 patients (88.8\%). Six patients were incised with scalp and drained. Six patients underwent mastectomy.
\end{abstract}

\section{Keywords}

Idiopathic Granulomatous Mastitis, Incidence and Experience, Descriptive 
Retrospective Study

\section{Introduction}

Idiopathic granulomatous mastitis (MGI) is a chronic inflammatory disease of the breast, benign, noninfectious, uncommon and difficult to diagnose. It was described in 1972 by Kessler and Wolloch [1]. It is characterized by chronic granulomatous inflammation of the lobes without necrosis and its clinical signs and symptoms can be so variable ranging from pain, fistula and secretion to the presence of a mass or tumor of the breast.

\subsection{Mechanisms and Pathophysiology}

Granulomatous mastitis is an idiopathic condition for which it has been proposed several mechanisms including autoimmune [2], infectious [3] processes and local reactions to chemical secretions [4]. However, the favorable responses to steroids in studies such as that of Ogura et al. [5] and Kazuhisa et al. [6] where MGI is classified as related and not related to Immunoglobulin 4 (IgG4), the consistent finding of negative cultures [7], as well as their association with other rheumatic diseases support an autoimmune etiology.

\subsection{Clinical Presentation}

MGI can mimic two very common breast diseases, breast cancer and breast abscess [8]. Most of the patients are of reproductive age, have a recent history of pregnancy and breastfeeding in the last 5 years [8]. However, cases have been reported in patients as young as 11 years [9] [10] to 80 years of age [11] [12].

It presents as a palpable unilateral mass, accompanied by abscesses that after culturing them in media for bacteria, mycobacteria and fungi are negative (aseptic cultures), although it constitutes a chronic inflammation of the gland, it can present local or systemic erythema (Figure 1, Figure 2), changes in the skin with retraction of the nipple or fixed to the pectoral muscle 7. MGI can occur in any quadrant of the breast, except in the subaerolar region [8].

\subsection{Diagnosis}

The diagnosis of this disease is reached after ruling out other diseases. Multiple biopsy techniques are used in the diagnosis of MGI. Fine needle aspiration biopsy is the least invasive method [13] [14], but it can lead to confusing results that often require confirmation by open biopsy. Open excisional biopsy is used with a good success rate, considering it the safest method to rule out malignancy [13]. The histological characteristic of the disease describes the presence of granulomas surrounded by stroma with giant cells, lymphocytes, plasma cells and neutrophils (Figure 3 ). However, histological features are also a challenge. In a study published by Lacambra et al. [7] comparing the histological differences 


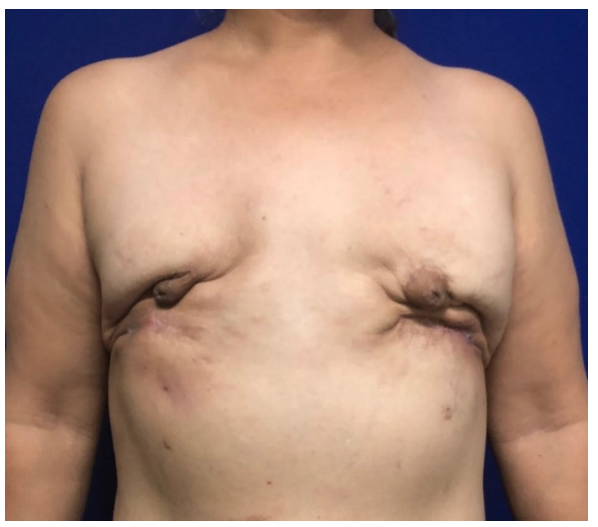

Figure 1. Severe bilateral case of recurrent Idiopathic Granulomatous Mastitis in a 33 years old female unsuccessfully treated with multiple pharmacological therapies and surgical resections along 10 years of evolution.

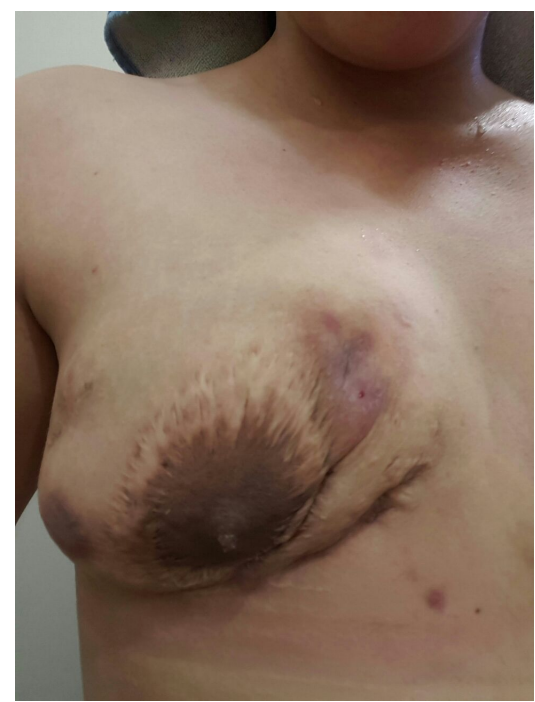

Figure 2. Image taken from: Binesh F. Idiopathic Granulomatous Mastitis, a Clinicopathological Review of 22 Cases. Journal of Clinical \& Experimental Pathology. 2014; 04(02).
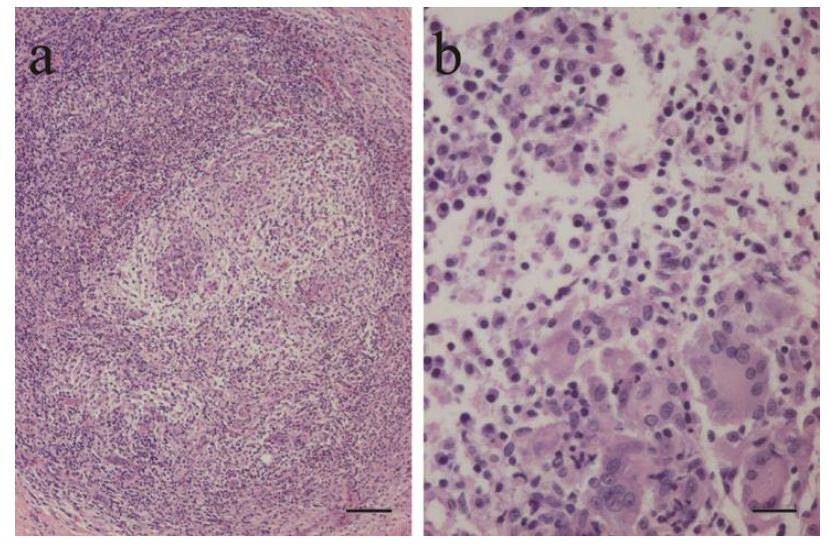

Figure 3. Histological findings showing granulomatous inflammation of the lobes without caseous necrosis and the presence of multinucleated giant cells and plasma cells. (Image taken from Kazuhisa Akahane et al. (2013). Therapeutic strategy for granulomatous lobular mastitis: a clinicopathological study of 12 patients. Nagoya J. Med. Sci. 75:193-200.) 
between Idiopathic Granulomatous Mastitis (33 cases) and Tuberculous Mastitis (29 cases), the group with tuberculous mastitis showed significantly more fibrosis, eosinophils and necrosis, while the MGI group showed significantly more plasma cells, so the diagnosis, although confirmed histologically, required other studies that rule out infectious etiology or foreign body reaction.

\subsection{Treatment}

There is no unification of criteria regarding treatment. Extensive surgical resections have been proposed, including total or partial mastectomy, curettage, surgical drainage, punctures, mechanical washes, as well as conservative treatments such as the use of anti-inflammatories, antibiotics and steroids combined with immunosuppressants.

Treatment with steroids was first proposed by Dehetrogh et al. [8] [15] and its efficacy in the treatment of MGI has been reported in several studies [5] [6] [8]. The steroid dose should start at $1 \mathrm{mg} / \mathrm{kg} /$ day and decrease gradually according to the clinical response [16]. Some patients may present hyperglycemia as an effect of high doses of prednisone [8], so the use of steroid-sparing agents such as methotrexate or azathioprine provides options that may facilitate the decrease of steroids [8] [17] [18]. Patients with a mass or pseudotumor equivalent to $20 \%$ $50 \%$ of the size of the breast that shows failure in medical treatment or intolerance to steroids are candidates for treatment with therapeutic mastectomy techniques [19]. Frequently, surgical management of MGI leads to disfigurement of the breast. However, the reconstruction of this must be a secondary objective, since the recurrence of the disease has occurred up to 24 months after the remission of symptoms. In a study conducted by Binesh et al., the findings demonstrated that surgical treatment is not always effective and is associated with a high recurrence rate [13] ranging from $5 \%$ to $50 \%$ [8] [12] [20].

\section{Materials and Methods}

We performed a descriptive retrospective study of the records from a Pemex Hospital in Mexico city for patients diagnosed with Idiopathic Granulomatous Mastitis histopathologically over a twelve year period between January 2005 and December 2017. During this period 9 women were diagnosed with IGM and treated. All of the women underwent a clinical breast examination to identify palpable lumps, skin thickening or axillary lymphadenopathy and breast pain. Definitive diagnosis was obtained by biopsy or surgical excision. The parameters assessed included age of patient, systemic and local symptoms and histological findings of inflammatory cells, granulomas, necrosis, multinucleated giant cells, fibrosis and calcifications. We included data and correlations in clinical presentation, comorbidities and treatment of patients with IGM in this hospital. We performed a descriptive analysis using central tendency measures.

\section{Results}

In a period of time between January 2005 and December 2017 (12 years), we in- 
cluded a total of 9 female patients. All of them have had histopathological findings consistent with granulomatous mastitis.

These patients were studied in a retrospective way after we analyzed their clinical presentation, evolution, histology and management of their disease. The age range was 25 - 83 years old (mean 50.8 yrs). Just one patient (11.1\%) has had a baby and recent lactation history. And another patient (11.1\%) had taken oral contraceptives. In 4 patients (44.4\%) had the right side affected, three patients (33.3\%) had the left side affected and 2 patients (22.2\%) have had a bilateral presentation. In 3 patients (33.3\%) we observed more than one affected quadrant and 4 patients (44.4\%) had a diffuse presentation on image, Table 1.

The most common symptom was breast pain in 9 patients $(100 \%)$ and the most common sign was the presence of a nodule, 8 patients $(88.8 \%)$. We observed erythema in 7 cases (77.7\%) followed by abscess, edema and pus secretion in 6 patients $(66.6 \%)$. The less frequent sign was nipple retraction in 2 patients (22.2\%) and axillary adenopathies in 3 patients (33.3\%).

The most frequent treatment was the use of a combination of NAIDs and antibiotic in 8 patients $(88.8 \%)$. We only treated one patient combining NSAIS and immunosuppressant. And another one was treated using antituberculosis drugs without confirming mycobacteria in culture. Regarding the surgical management of this disease, 6 patients were incised with scalp and drained. 6 patients underwent mastectomy, 2 of them had bilateral mastectomy and another 2 were treated by quadrantectomy.

In the follow-up the mean of office consultations were 51.3 with mean of 6.2 surgical interventions in between. The mean of hospitalizations was 5.3 and the mean of hospital stay was 42.3 days. One particular patient accumulated a total of 270 days of stay, which was the longest period of time reported in all cases. Five patients had no signs and symptoms after a 5 year period of treatment.

\section{Discussion}

Idiopathic Granulomatous Mastitis (IGM) is characterized by the presence of granulomatous necrotizing lobulitis of unknown etiology [21] [22]. We can find

Table 1. Characteristics of patients with idiopathic granulomatous mastitis.

\begin{tabular}{cccccccccc}
\hline PatientsNo. & 1 & 2 & 3 & 4 & 5 & 6 & 7 & 8 & 9 \\
\hline Age & 43 & 83 & 74 & 65 & 33 & 70 & 25 & 36 & 29 \\
Parity & - & - & - & - & - & - & - & + & - \\
Breast fed & - & - & - & - & - & - & - & + & - \\
Contraceptives & - & - & - & - & - & - & + & - & - \\
Affected breast & R & L & R & L & B & R & Di & L & B \\
Affected quadrant & 1 & 1 & 1 & 1 & $>1$ & 1 & $>1$ & 1 & $>1$ \\
Distribution & Lo & Lo & Lo & Lo & Di & Di & Di & Lo & Di \\
\hline
\end{tabular}

-: Negative; +: Positive; R: Right; L: Left; Lo: Local; Di: Difuse. 
this entity in 24\% of all the breast inflammatory pathologies [23]. Until 1999 we can identify 120 reported cases [24] in international literature since the complete description of the illness performed by Kessler y Wollock in 1972 [1]. Recently a study performed in 22 breast center in Turkey was published [25], that included 720 patients diagnosed with IGM treated between January 2011 and March 2016 and supports greatest knowledge and suspicion of this disease.

The mean age of the patients studied in our work presented a mean age of presentation, of 50.8 years old. whilst international literature report a mean age of presentation under 50 years [21], with a mean age of 36.5 years old [22] [26]. It's also reported that up to $11.1 \%$ of the patients with this disease have an association with contraceptive use and breastfeeding, a different study reported that one third of the patients with this disease presented a history of oral contraceptives use [21], however, the greatest series of cases [25] reported no association between the disease and the use of contraceptives or breastfeeding.

In our study we observed breast pain (mastalgia) in 100\% and unilateral nodule present in $88.8 \%$ of the patients as the most frequent symptoms of the disease, similar to international literature that reports the presence of unilateral mass in 57\% and mastalgia in 33\% [22] [25] [26]. Axillary limphadenopathies and nipple retraction were the less frequent signs observed as many other studies has reported [22] [26], however, some of the complications that may be developed include abscesses, fistula and chronic discharge of pus or other secretion [21] [27].

We found bilateral affection in $22 \%$ of this group of patients, $44 \%$ had right breast presentation and 33\% in left side. We performed a comparison with international reports where they describe a 3\% - 5\% bilateral affection and 38\% and 58\% right and left affection respectively [25] [26].

During the patients files review we observed the clinical approach of the disease including a number of lab and cabinet studies such as FNA-biopsies ultrasound guided, ultrasound images, mammography, CT scan and MRI. These last two had reported malignancy findings most frequently. Some authors suggest to perform sequenced studies using FNA-biopsy or excisional biposy in a prior way until undergoing mastectomy in order to avoid mistaken diagnosis and rule out carcinoma [21]. Negative cultures using different types of stains, Polymerase Chain Reaction (PCR), anti rheumatoid antibodies also were made to supports idiopathic nature of granulomatous mastitis.

We suspected IGM ruling out differential diagnosis and the presence of histopathological findings in every specimen analyzed. These findings included granulomatous inflammation with diffuse obliteration of lobar architecture, likewise inflammatory infiltrate with leucocytes, polymorphonuclear cells, plasmatic cells, epithelioid cells and Langhans giant cells in the granulomas. We observed that the term granulomatous mastitis is overused by pathological findings compatible with inflammatory response [22]. In fact 31 patients have been diagnosed with granulomatous mastitis; however, only 9 patients were compatible with this disease over the clinical presentation and following, clinical criteria suggesting that 
we need to overlook the whole presentation of the disease in order to make an accurate diagnosis.

Antibiotics are the most commonly used agents in the treatment of IMG as well as systemic steroids [25]. Authors recommend to suspend the use of antibiotics once the diagnosis is confirmed since is not about an infectious disease. Prednisone $0.8 \mathrm{mg} / \mathrm{kg} /$ day divided in two doses is the initial dose recommended [28].

Other authors reported oral steroids as treatment of choice (39\%) followed by antibiotics in $37 \%$, a combination of these two in $13 \%$ and methotrexate intake in $1 \%$ of the patients [25]. In our institution we used the combined form of treatment consisting in oral NSAIDs and antibiotics in $88.8 \%$, steroid and immunosuppressant agents in $11.1 \%$ and anti-tuberculous agents in $11.1 \%$ of the patients.

The results involving surgical treatment shows better outcomes when compared versus steroid therapy with decrease recovery time and lower recurrence rates. This is the reason why some authors recommend the steroid treatment option for recurrent disease only. Performing drainage in the presence of abscess has the highest recurrence reported by $50 \%$ of the patients [28], so they suggest performing initial extended excision rather than drainage.

Carrying out this study we realized that we have had higher rates of surgical management of the disease in our Institution than the rates reported by international literature. We observed that $66 \%$ of the patients underwent mastectomy, $22 \%$ extended excision and only $12 \%$ were treated by drainage. None of our patients received only medical management for their disease. In recent studies medical treatment is used in $36 \%$ as a single therapy. The surgical intervention was reported only by $8 \%$. The combination of these two in $56 \%$ being the extended excision the most commonly surgical treatment used in $69 \%$, followed by drainage in $29 \%$ and mastectomy in $1.3 \%$ [21].

The recurrence rate is described to be up to $38 \%$ of the patients. So they recommend follow up after surgical treatment or indicate corticosteroids therapy [21].

\section{Conclusion}

Granulomatous Mastitis is a chronic benign disease with high recurrence rate and it is mandatory that the diagnosis is made in order to avoid mistaken carcinoma diagnosis. We need to correlate clinical evolution and histopathological findings in order to obtain an accurate diagnosis. The current treatment of this particular disease still remains controversial. We observed a different patient response in the current treatment options. However, we believe that the use of a combination of immunosuppressant and NSAIDs represents an open-door in our institution because of the strong information with sustaining evidence that supports efficacy and effectiveness of this treatment option. We also analyzed the days the patients were hospitalized and the number of surgical interventions 
performed in every case.

\section{Conflicts of Interest}

The authors declare no conflicts of interest regarding the publication of this paper.

\section{References}

[1] Kessler, E. and Wolloch, Y. (1972) Granulomatous Mastitis: A Lesion Clinically Simulating Carcinoma. American Journal of Clinical Pathology, 58, 642-646.

https://doi.org/10.1093/ajcp/58.6.642

[2] Brown, K. and Tang, P. (1979) Postlactationaltumoral Granulomatous Mastitis: A Localized Immune Phenomenon. The American Journal of Surgery, 138, 326-329. https://doi.org/10.1016/0002-9610(79)90397-0

[3] Fletcher, A., Magrath, I., Riddell, R. and Talbot, I. (1982) Granulomatous Mastitis: A Report of Seven Cases. Journal of Clinical Pathology, 35, 941-945. https://doi.org/10.1136/jcp.35.9.941

[4] Rowe, P.H. (1984) Granulomatous Mastitis Associated with a Pituitary Prolactinoma. British Journal of Clinical Practice, 38, 32-34.

[5] Ogura, K., Matsumoto, T., Aoki, Y., Kitabatake, T., Fujisawa, M. and Kojima, K. (2010) IgG4-Related Tumour-Forming Mastitis with Histological Appearances of Granulomatous Lobular Mastitis: Comparison with Other Types of Tumour-Forming Mastitis. Histopathology, 57, 39-45. https://doi.org/10.1111/j.1365-2559.2010.03581.x

[6] Akahane, K., et al. (2013) Therapeutic Strategy for Granulomatous Lobular Mastitis: A Clinicopathological Study of 12 Patients. Nagoya Journal of Medical Science, 75, 193-200.

[7] Lacambra, M., Thai, T., Lam, C., Yu, A., Pham, H., Tran, P., et al. (2011) Granulomatous Mastitis: The Histological Differentials. Journal of Clinical Pathology, 64, 405-411. https://doi.org/10.1136/jcp.2011.089565

[8] Patel, et al. (2009) Idiopathic Granulomatous Mastitis: Case Reports and Review of Literature. Journal of General Internal Medicine, 25, 270-273. https://doi.org/10.1007/s11606-009-1207-2

[9] Bani-Hani, K., Yaghan, R., Matalka, I. and Shatnawi, N. (2004) Idiopathic Granulomatous Mastitis: Time to Avoid Unnecessary Mastectomies. The Breast Journal, 10, 318-322. https://doi.org/10.1111/j.1075-122X.2004.21336.x

[10] Katz, U., Molad, Y., Ablin, J., et al. (2007) Chronic Idiopathic Granulomatous Mastitis. Annals of the New York Academy of Sciences, 1108, 603-608.

https://doi.org/10.1196/annals.1422.063

[11] Lai, E., Chan, W., Ma, T., Tang, A., Poon, C. and Leong, H. (2005) The Role of Conservative Treatment in Idiopathic Granulomatous Mastitis. The Breast Journal, 11, 454-456. https://doi.org/10.1111/j.1075-122X.2005.00127.x

[12] Asoglu, O., Ozmen, V., Karanlik, H., Tunaci, M., Cabioglu, N., Igci, A., et al. (2005) Feasibility of Surgical Management in Patients with Granulomatous Mastitis. The Breast Journal, 11, 108-114. https://doi.org/10.1111/j.1075-122X.2005.21576.x

[13] Binesh, F. (2014) Idiopathic Granulomatous Mastitis, a Clinicopathological Review of 22 Cases. Journal of Clinical \& Experimental Pathology, 4, 157. https://doi.org/10.4172/2161-0681.1000157 
[14] Agale, S.V., Momin, Y.A. and Khan, W.A. (2009) Cytology of Idiopathic Granulomatous Mastitis: A Report of a Case Masquerading as Carcinoma. Bombay Hospital Journal, 51, 105.

[15] DeHertogh, D., Rossof, A., Harris, A. and Economou, S. (1980) Prednisone Management of Granulomatous Mastitis. New England Journal of Medicine, 303, 799-800. https://doi.org/10.1056/NEJM198010023031406

[16] Azlina, A., Ariza, Z., Arni, T. and Hisham, A. (2003) Chronic Granulomatous Mastitis: Diagnostic and Therapeutic Considerations. World Journal of Surgery, 27, 515-518. https://doi.org/10.1007/s00268-003-6806-1

[17] Kim, J., Tymms, K. and Buckingham, J. (2003) Methotrexate in the Management of Granulomatous Mastitis. ANZ Journal of Surgery, 73, 247-249. https://doi.org/10.1046/j.1445-1433.2002.02564.x

[18] Raj, N. (2004) Rheumatologists and Breasts: Immunosuppressive Therapy for Granulomatous Mastitis. Rheumatology, 43, 1055-1056. https://doi.org/10.1093/rheumatology/keh246

[19] Ahmed, Y. and Abd El Maksoud, W. (2016) Evaluation of Therapeutic Mammoplasty Techniques in the Surgical Management of Female Patients with Idiopathic Granulomatous Mastitis with Mild to Moderate Inflammatory Symptoms in Terms of Recurrence and Patients' Satisfaction. Breast Disease, 36, 37-45. https://doi.org/10.3233/BD-150198

[20] Schelfout, K., Tjalma, W.A., Cooremans, I.D., Coeman, D.C., Colpaert, C.G. and Buytaert, P.M. (2001) Observations of an Idiopathic Granulomatous Mastitis. European Journal of Obstetrics \& Gynecology and Reproductive Biology, 97, 260-262. https://doi.org/10.1016/S0301-2115(00)00546-7

[21] Imoto, S., Kitaya, T., Kodama, T., Hasebe, T. and Mukai, K. (1997) Idiopathic Granulomatous Mastitis: Case Report and Review of the Literature. Japanese Journal of Clinical Oncology, 27, 274-277. https://doi.org/10.1093/jjco/27.4.274

[22] Vinayagam, R., Cox, J. and Webb, L. (2009) Granulomatous Mastitis: A Spectrum of Disease. Breast Care, 4, 251-254. https://doi.org/10.1159/000229541

[23] Kayahan, M., Kadioglu, H. and Muslumanoglu, M. (2012) Management of Patients with Granulomatous Mastitis: Analysis of 31 Cases. Breast Care, 7, 226-230. https://doi.org/10.1159/000337758

[24] Memis, A., Bilgen, I., Ustun, E.E., et al. (2002) Granulomatous Mastitis: Imaging Findings with Histopathologic Correlation. Clinical Radiology, 57, 1001-1006. https://doi.org/10.1053/crad.2002.1056

[25] Uysal, E., Soran, A. and Sezgin, E. (2017) Factors Related to Recurrence of Idiopathic Granulomatous Mastitis: What Do We Learn from a Multicentre Study? ANZ Journal of Surgery, 88, 635-639. https://doi.org/10.1111/ans.14115

[26] Akcan, A., Akyndiz, H., Deneme, M., et al. (2006) Granulomatous Lobular Mastitis: A Complex Diagnostic and Therapeutic Problem. World Journal of Surgery, 30, 1403-1409. https://doi.org/10.1007/s00268-005-0476-0

[27] Going, J.J., Anderson, T.J., Wilkinson, S. and Chetty, U. (1987) Granulomatous Lobular Mastitis. Journal of Clinical Pathology, 40, 535-540. https://doi.org/10.1136/jcp.40.5.535

[28] Ozmen, V., Cantürk, Z., Celik, V., et al. (2012) Breast Disease. Federation of Breast Diseases Society, Gunes Medical Publishing, Ankara. 